\title{
Síntomas neurológicos motores de inicio súbito en el síndrome de Leriche
}

\author{
Sudden onset motor neurological symptoms in Leriche's
}

\author{
syndrome
}

\author{
Valentina Patiño-Pérez ${ }^{1}$ ORCID , Juan Camilo Tobar-Solarte ${ }^{2}$ ORCID, Richard Fernando Imbachi ${ }^{3}$ ORCID, \\ María Virginia Pinzón-Fernándež ORCID
}

Fecha correspondencia:

Recibido: junio 23 de 2020.

Revisado: marzo 1 de 2021.

Aceptado: marzo 9 de 2021.

Forma de citar:

Patiño-Pérez V, Tobar-Solarte JC, Imbachi RF, Pinzón-Fernández MF. Síntomas neurológicos motores de inicio súbito en el síndrome de Leriche. Rev CES Med. 2021; 35(1): 68-74.

Open access

(C) Derecho de autor

Licencia creative commons

Ética de publicaciones

Revisión por pares

Gestión por Open Journal System DOl: http://dx.doi.org/10.21615/

cesmedicina.35.1.8

ISSN 0120-8705

e-ISSN 2215-9177

Comparte

F日G. $898 R^{\circ}$ 마

\section{Resumen}

El síndrome de Leriche es una enfermedad poco frecuente, con gran morbilidad y mortalidad, que ocurre debido a oclusión de las ramas principales de la aorta abdominal por debajo de la bifurcación de las arterias renales. Clínicamente, se caracteriza por la tríada de claudicación, impotencia y disminución de los pulsos femorales. El diagnóstico se confirma por ecografía Doppler abdominal o angiografía por tomografía computarizada y el tratamiento se basa en el restablecimiento del flujo por debajo del nivel de la oclusión. Se presenta el caso de un paciente con cuadro clínico atípico de dolor lumbar, paraparesia y livideces de miembros inferiores de inicio súbito, con rápido deterioro de su estado general que requirió intervención endovascular urgente. En esta ocasión, los síntomas neurológicos pueden ser explicados por la reducción del flujo sanguíneo de la arteria espinal magna, que en el $25 \%$ de la población se origina en L1 o por debajo de ella y puede generar isquemia de la médula espinal. La presentación atípica de este reporte lo convierte en un desafío clínico de gran importancia.

Palabras clave: Síndrome de Leriche; Aorta Abdominal; Arteriopatías oclusivas; Manifestaciones neurológicas; Enfermedades vasculares periféricas.

\footnotetext{
Abstract

Leriche syndrome is a rare disease with high morbidity and mortality that occurs due to occlusion of the main branches of the abdominal aorta below the bifurcation of the renal arteries. Clinically, it is characterized by the triad of claudication, impotence and decreased femoral pulses. Diagnosis is confirmed by abdominal Doppler ultrasound or computed tomography angiography and treatment is based on restoration of arterial flow below the level of occlusion. We present the case of a patient with an atypical clinical presentation of sudden onset of low back pain, paraparesis and lividity of the lower limbs, with rapid deterioration of his general condition that required urgent endovascular intervention. On this occasion, the neurological symptoms can be explained by reduced blood flow in the magna spinal artery, which in 25\% of the population originates at or below L1 and can generate spinal cord ischemia. The atypical presentation of this report makes it a clinical challenge of great importance.
} 
Enero - abril de 2021 - Pág 69

\section{Sobre los autores:}

1. Estudiante de Medicina. Grupo de Investigación en Salud Universidad del Cauca.

2. Médico, Hospital Susana López de Valencia, UMI.Grupo de Investigación en Salud, Universidad del Cauca.

3. Especialista en Medicina Interna. Hospital San JoséClínica la Estancia. Docente Universidad del Cauca, Grupo de Investigación en Salud.

\section{MSP- PhD. Docente} Universidad del Cauca, Grupo de Investigación en Salud.
Una presentación menos frecuente del síndrome de Leriche, y en ocasiones subdiagnosticada, es la paraplejia aguda debida a la reducción del flujo sanguíneo de la arteria de Adamkiewicz, que causa isquemia en la médula espinal.
Keywords: Leriche Syndrome; Aorta Abdominal, Arterial Occlusive Diseases; Neurological Symptoms; Peripheral Vascular diseases.

\section{Introducción}

El síndrome de Leriche, también conocido como enfermedad oclusiva aortoilíaca, es una entidad en la que se presenta una oclusión de las ramas principales de la aorta abdominal por debajo de la bifurcación de las arterias renales, comprometiendo arterias ilíacas, femorales y tibiales de ambos miembros inferiores (1). Es consecuencia de una lesión de la pared arterial inducida por arterioesclerosis y conduce a la alteración de los tres factores involucrados en la tríada de Virchow: el daño endotelial, la trombosis (2) y menos frecuentemente la embolia (3). Puede presentarse de forma subaguda o súbita, la cual se asocia con alta tasa de mortalidad $(4,5)$.

Clínicamente, se caracteriza por la tríada de claudicación, impotencia y disminución de los pulsos femorales (6-8). Una presentación menos frecuente, y en ocasiones subdiagnosticada, es la paraplejia aguda debida a la reducción del flujo sanguíneo de la arteria de Adamkiewicz, que causa isquemia en la médula espinal. Sin embargo, es un verdadero desafío clínico diferenciar entre el origen vascular y el neurogénico de la paraplejía dolorosa de inicio agudo; por tal razón, el conocimiento de esta enfermedad, asociado a una alta sospecha clínica y un exhaustivo examen físico, hace la diferencia en los enfoques diagnóstico y terapéutico $(3,9)$.

El objetivo de este artículo es presentar el caso clínico de un paciente quién consultó a urgencias con déficit neurológico motor en miembros inferiores, de inicio súbito, secundario a un síndrome de Leriche, siendo una manifestación poco común de esta entidad.

\section{Presentación del caso}

Un paciente masculino de 61 años consultó por dolor en región lumbar. Ingresó a un servicio de urgencias de tercer nivel en la ciudad de Popayán, Colombia, con cuadro clínico de aproximadamente 24 horas de evolución consistente en dolor lumbar de inicio súbito, progresivo en intensidad, irradiado a miembros inferiores y asociado a hipotonía, disestesias, parestesias simétricas y paraparesias. Posterior a su ingreso, el paciente se deterioró rápidamente presentando incremento del dolor lumbar, franca paraplejia con ausencia de pulsos femorales, poplíteos y pedios, llenado capilar prolongado, frialdad y livideces en miembros inferiores (imagen 1). Al momento de su llegada al servicio el paciente presentaba adecuado control de esfínteres; sin embargo, por su deterioro clínico se decidió pasar una sonda vesical.

Adicionalmente, reportaba antecedentes de diabetes mellitus tipo 2, hipertensión arterial, miocardiopatía dilatada de origen mixto: isquémico-esclero-hipertensiva con fracción de eyección disminuida (28 \%), trombosis venosa profunda en miembro inferior derecho hace cinco años y tabaquismo hasta hace 20 años (no se pudo precisar por historia clínica el índice paquete/año). Al momento se encontraba en tratamiento farmacológico con sacubitril/valsartán, carvedilol, digoxina y había suspendido warfarina tres meses antes de su ingreso. 
Enero - abril de 2021 - Pág 70

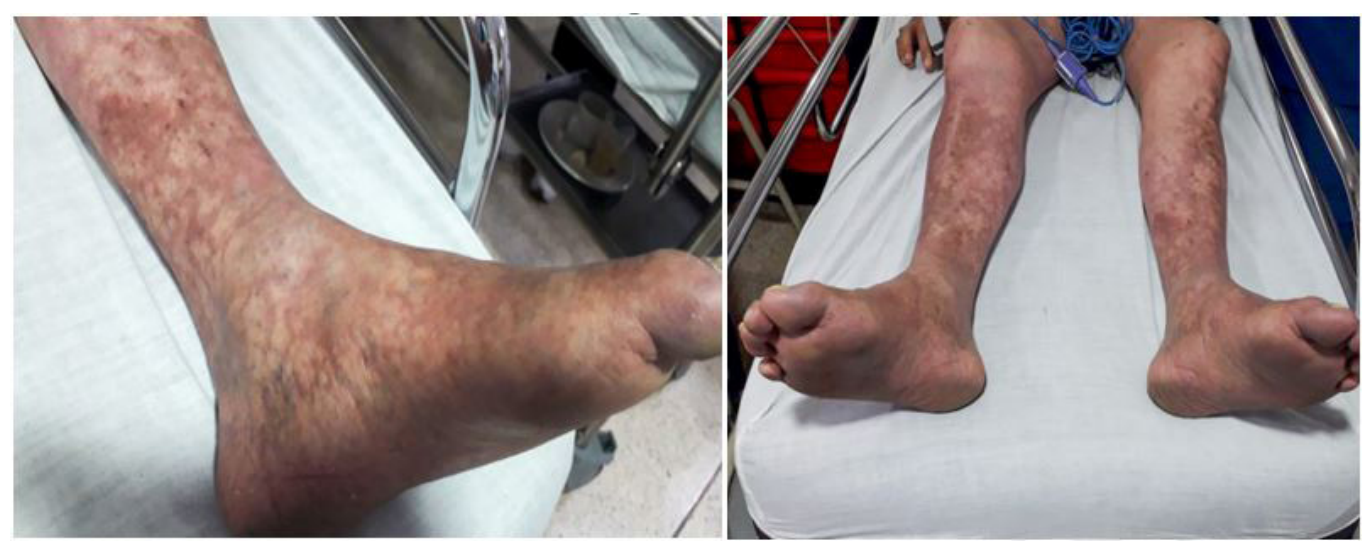

Imagen 1. Livideces bilaterales asociadas a cianosis

El paciente ingresó con cuadro clínico de 24 horas de evolución de dolor lumbar de inicio súbito, progresivo en intensidad, irradiado a miembros inferiores y asociado a hipotonía, disestesias, parestesias simétricas y paraparesias. Posteriormente, presentó rápido deterioro por incremento del dolor lumbar, franca paraplejia con ausencia de pulsos femorales, poplíteos y pedios, llenado capilar prolongado, frialdad y livideces en miembros inferiores).
Después de su descompensación, le fue realizada una angiotomografía de abdomen que evidenció oclusión aórtica por gran trombo a nivel infra-renal, con interrupción del flujo hacia las arterias ilíacas y sus ramificaciones, además de escaso medio de contraste endoluminal en arterias femorales por llenado mediante circulación colateral (imágenes 2 y 3 ).

Debido a los hallazgos fue llevado de emergencia a sala de angiografía para manejo endovascular, en donde se le realizó trombectomía mecánica de aorta abdominal, arterias ilíacas y femorales, además de trombólisis intraarterial con alteplase, logrando recuperación del flujo distal. En el post operatorio inmediato, el paciente presentó dos episodios de paro cardíaco con reanimación cardiopulmonar exitosa; fue llevado luego a la unidad de cuidados intensivos donde presentó falla multiorgánica con bloqueo AV completo, requiriendo soporte eléctrico (marcapasos), vaso activo e inotrópico sin respuesta favorable y siete horas más tarde falleció.
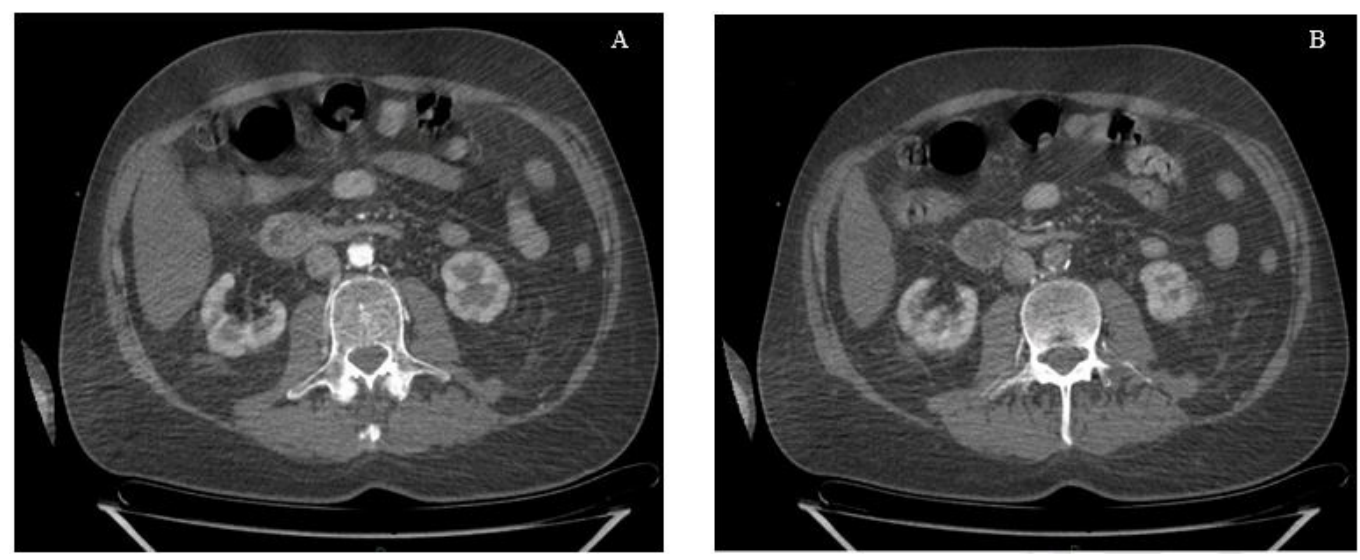

Imagen 2. Angiotomografía abdominal. A: corte axial con evidencia de medio de contraste en la aorta abdominal. B: corte axial con interrupción abrupta del flujo de medio de contraste 

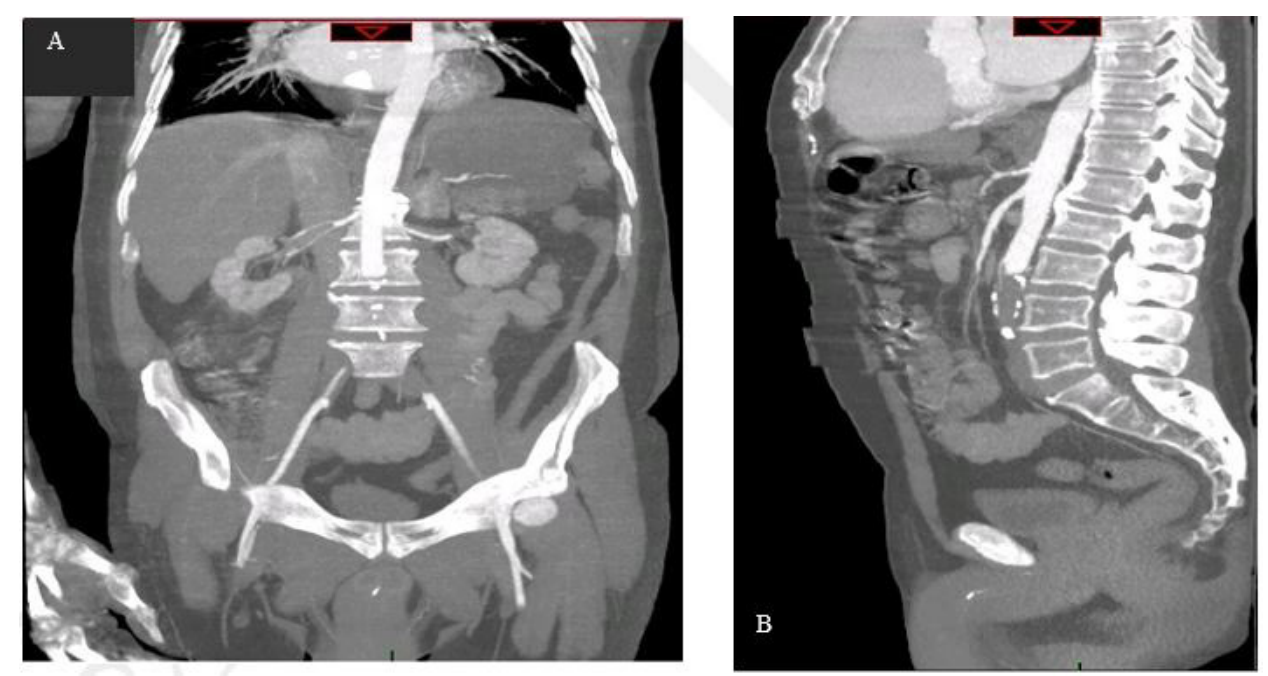

Imagen 3. Angiotomografía de abdomen. A: corte coronal, evidencia interrupción del flujo de la aorta abdominal por debajo de las arterias renales. B: corte sagital, interrupción del flujo de la aorta abdominal a nivel de L3 aproximadamente.

Aunque la triada de claudicación, impotencia y disminución de los pulsos femorales está presente en el $73 \%$ de los pacientes con síndrome de Leriche, también se describen otros síntomas menos frecuentes como entumecimiento o dolor intenso en región lumbar, paraparesia 0 paraplejia de miembros inferiores, además de palidez o apariencia moteada de los mismos, identificados en el paciente reportado.

\section{Discusión}

La enfermedad aortoilíaca oclusiva completa consiste en la obstrucción total de la aorta abdominal y sus ramas principales, como las arterias ilíacas, femorales e incluso tibiales (1), siendo una variante rara de la enfermedad oclusiva aterosclerótica clásica, descrita por primera vez en 1814 por Robert Graham; sin embargo, solo hasta 1940 el cirujano francés René Leriche logró intervenir quirúrgicamente un paciente de 29 años, en quien describió la triada clásica de claudicación, impotencia y disminución de los pulsos femorales, por lo cual la enfermedad recibió el nombre de síndrome de Leriche $(10,11)$.

El síndrome de Leriche tiene una incidencia, estimada de 0,15 \% en toda la población mundial (12), con una mortalidad que varía entre el 25 y el $75 \%(4,5)$; otros autores, registran prevalencias en la población entre 25-65 años del 0,7 \% en mujeres y 1,3 $\%$ en hombres (13). La etiología más común es la trombosis en lugar de la embolia (14); se presenta más frecuentemente en hombres, entre la tercera y sexta década de vida $(7,15)$, como en el caso descrito.

Los factores de riesgo comúnmente reportados son la hipertensión arterial, hiperlipidemia, diabetes mellitus, edad, raza, historia familiar y tabaquismo $(2,7,15)$, este último presente hasta en el $90 \%$ de los casos (4). Otros factores de riesgo menos comunes son los defectos de desarrollo del crecimiento aórtico, exposición a radiación, infección congénita por rubéola, aortitis lúetica, arteritis de Takayasu, fibrosis retroperitoneal y enfermedades autoinmunes como lupus eritematoso sistémico, síndrome antifosfolípido primario o secundario (16).

Por otro lado, aunque la triada de claudicación, impotencia y disminución de los pulsos femorales está presente en el $73 \%$ de los pacientes con síndrome de Leriche (4); también se describen otros síntomas menos frecuentes como entumecimiento o dolor intenso en región lumbar, paraparesia o paraplejia de miembros inferiores, además de palidez o apariencia moteada de los mismos, identificados en el paciente reportado $(5,9,15)$. 
Este caso es de gran importancia dada su presentación atípica, convirtiéndolo en un verdadero desafío clínico al requerir establecer la diferencia entre el origen vascular o el neurogénico de la paraplejía dolorosa de inicio agudo.
Los síntomas neurológicos son explicados por la reducción del flujo sanguíneo de la arteria espinal magna o de Adamkiewicz, que irriga las raices espinales que forman el plexo lumbosacro; adicionalmente, esta arteria tiene un origen muy variable a lo largo de la médula espinal pudiendo originarse entre T5 y T8 en el $15 \%$ de los casos, entre T9 y T12 en el $60 \%$ y en L1 en el $25 \%$ restante, lo que puede desencadenar isquemia de la médula espinal (17). Este caso es de gran importancia dada su presentación atípica, convirtiéndolo en un verdadero desafío clínico al requerir establecer la diferencia entre el origen vascular o el neurogénico de la paraplejía dolorosa de inicio agudo $(3,7,13)$.

El paciente presentó dolor lumbar intenso, asociado a paraparesia de inicio súbito que evolucionó a paraplejía con livideses de miembros inferiores, además de ausencia de pulsos en los mismos, que son el sello distintivo del síndrome de Leriche $(2,13)$. La sospecha clínica temprana permitió dar manejo oportuno y confirmar el diagnóstico mediante angiografía por tomografía computarizada que, junto con la ecografía Doppler abdominal, resultan los métodos de elección para la confirmación de la oclusión aorto-ilíaca (15).

Respecto al tratamiento, generalmente es quirúrgico y consiste en la recuperación del flujo distal a la oclusión (4). Las intervenciones endovasculares o las reparaciones quirúrgicas abiertas son los tratamientos clásicos (19); así pues, la revascularización por medio de intervenciones endovasculares ha reemplazado a la cirugía de derivación aortofemoral como terapia de primera línea, motivo por el cual el paciente fue llevado a terapia endovascular.

No obstante, en los casos donde las técnicas endovasculares no tienen éxito o son inapropiadas, el bypass aorto-femoral ha mostrado mejores resultados a largo plazo, pudiendo considerase el estándar de oro, debido a que cuenta con excelentes tasas de permeabilidad (entre el 72 y $90 \%$ a los 10 años) $(4,15,19)$.

Aunque las tasas de permeabilidad primaria a largo plazo con el bypass abierto son superiores a las de la intervención endovascular (4), la reconstrucción abierta tiene mayor morbimortalidad, hospitalización más prolongada y mayores costos, a diferencia de los procesos endovasculares. Por ello se ha sugerido que, incluso en casos complejos, debería intentarse una terapia endovascular antes de llevar al paciente a cirugía abierta (4). Esto basado en que casi todas las oclusiones aorto-ilíacas se pueden tratar con éxito por medio del enfoque endovascular (4) y que el pronóstico o la permeabilidad a cinco años han sido adecuadas (18).

Por otra parte, se reportan resultados favorables en pacientes sometidos a trombolisis intra-arterial local con posterior colocación de stent; sin embargo, no es una terapia estandarizada o avalada de uso rutinario $(20,21)$. Adicionalmente, no se encontró información sobre la utilidad de la trombolisis sistémica por vía intravenosa ni de la terapia anticoagulante inicial. No obstante, en la práctica clínica siempre se debera tener cuenta la fisiopatología trombo-oclusiva y evaluar los beneficios de iniciar dicho tratamiento.

Finalmente, el paciente evolucionó hacia la disfunción multiorgánica y muerte, probablemente a consecuencia del fenómeno de reperfusión, entidad que genera la mayor parte de las complicaciones no asociadas con trombosis aguda del stent o bypass (23) y que excede el propósito de este reporte de caso. 


\section{Conclusiones}

El sindrome de Leriche es una enfermedad aorto-ilíaca oclusiva completa, que se produce por trombosis local sobre una base de enfermedad aterosclerótica y menos frecuentemente por embolia. Su presentación clásica se define por la triada de claudicación, impotencia y disminución de los pulsos femorales, aunque en menor proporción se asocia con lumbalgia y síntomas neurológicos motores de miembros inferiores, de aparición súbita. Debido a su alta mortalidad, es importante la sospecha clínica temprana que permita un adecuado enfoque terapeútico para mejorar su pronóstico, teniendo en cuenta que a pesar del tratamiento quirúrgico o endovascular oportuno, existe un alto riesgo de muerte.

\section{Aspectos éticos}

Para la realización de este reporte de caso, se contó con el consentimiento informado del familiar del paciente.

\section{Bibliografía}

1. Muñoz Chaves JC, Solarte Pineda H, Imbachi R. Caso clínico de interés: síndrome de Leriche. Rev Colomb Salud Libr. 2016;11 (1):57-61.

2. Brown K, Gonzalez L. Leriche Syndrome. Eur PMC [Internet]. 2020; Available from: https://europepmc.org/books/NBK538248;jsessionid=923065688CC5E24ED0B-

Se ha sugerido que, incluso en casos complejos debería intentarse una terapia endovascular antes de llevar el paciente a una cirugía abierta. 8D9357B80F70E

3. Zankl AR, Blessing E, Volz HC, Krumsdorf U, Katus HA, Andrassy M. Neurological symptoms in acute Leriche 's syndrome. Clin Res Cardiol. 2010;99:459-62. doi. org/10.1007/s00392-010-0121-

4. Clair DG, Beach JM. Strategies for managing aortoiliac occlusions: access, treatment and outcomes. Expert Rev Cardiovasc Ther. 2015;13 (5):551-63. doi:

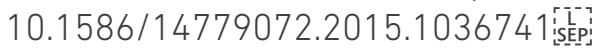

5. Quenzer F, Stillings J, Le J. Dead Legs: A Case of bilateral leg paralysis. Clin Pract Cases Emerg Med. 2017;1 (4):315-8.

6. Furui M, Hirata H, Kakii B, Uchino G, Asanuma M, Suzuki H, et al. Successful surgical treatment of an infected thoracoabdominal aneurysm accompanied with Leriche syndrome. Case Rep Surg. 2019;2019:1-4.

7. McCoy CE, Patierno S, Lotfipour S. Leriche syndrome presenting with multisystem vaso-occlusive catastrophe. West J Emerg Med. 2015;16 (4):583-6.

8. Kita K. Leriche syndrome (Aortoiliac occlusive disease). J Gen Fam Med. 2017;18 (5):297-8.

9. Mahendrakar SM, Sandhu HS, Khan AH, Loya YS. Leriche syndrome: Acute onset painful paraplegia of vascular origin with catastrophic consequences. J Clin Diagnostic Res. 2017;11 (5):22-3.

10. Lee W-J, Cheng Y-Z, Lin H-J. Leriche syndrome. Int J Emerg Med. 2008; (14):426-8. 
11. Jarcho S. Historical milestones coarctation of the aorta. Am J Cardiol. 1961;8 (2):264-9.

12. Depboylu BC, Harmandar B, Yazman S, Arslan K. Struggle in struggle: Surgical treatment of Leriche syndrome in a horseshoe kidney patient. Turkish J Thorac Cardiovasc Surg. 2019;27 (4):586-9.

13. Syndrome L. Diagnóstico por tomografía del Síndrome de Leriche: reporte de un caso clínico. 2013:36 (2):105-7.

14. Crawford JD, Perrone KH, Wong VW, Mitchell EL, Azarbal AF, Liem TK, et al. A modern series of acute aortic occlusion. J Vasc Surg [Internet]. 2014;59 (4):1044-50. Available from: http://dx.doi.org/10.1016/j.jvs.2013.10.080

15. An X, Fu R, Zhao Z, Ni X, Xiong C, Cheng X, et al. Leriche syndrome in a patient with acute pulmonary embolism and acute myocardial infarction: A case report and review of literature. BMC Cardiovasc Disord. 2020;20 (1):1-5.

16. Keller K, Beule J, Oliver Balzer J, Coldewey M, Munzel T, Dippold W, et al. A 56-yearold man with co-prevalence of Leriche syndrome and dilated cardiomyopathy: Case report and review. Wien Klin Wochenschr. 2014;126 (5-6):163-8.

17. Wikinski JA, Salgueiro C. La arteria de Adamkiewicz y su papel en la irrigación medular. Una actualización bibliográfica basada en internet. Rev Argent Anestesiol. 2003;61 (3):170-81.

18. Rodríguez SP, Sandoval F. Aortoiliac occlusive disease, a silent syndrome. BMJ Case Rep. 2019;12 (7):1-2.

19. Dellehunt RE, Manna B. Aortofemoral bypass. StatPearls. 2018 Jan 1;2:2332-42.

20. Nanto K, lida O, Fujihara M, Yokoi Y, Tomoi Y, Soga Y, et al. Five-year patency and its predictors after endovascular therapy for aortoiliac occlusive disease. J Atheroscler Thromb. 2019;26 (11):989-96.

21. Bean W, Rodan B, Thebault A. Leriche syndrome: treatment with streptokinase and angioplasty. Am J Roentgenol. 1985;144:1285-6.

22. Katzenschlager R, Ahmadi A, Koppensteiner R, Minar E, Stümpflen A UA. Leriche syndrome: treatment with local lysis and subsequent percutaneous transluminal angioplasty. Ehringer H Vasa. 1996;25 (2):180-3.

23. Raffan S. F. Síndrome de reperfusión: fisiopatología, prevención y manejo. Rev colomb anestesiol. 2000;28 (4):301-6. 